\title{
SEMIMODULAR FUNCTIONS AND COMBINATORIAL GEOMETRIES ${ }^{1}$
}

BY

\author{
HIEN QUANG NGUYEN
}

\begin{abstract}
A point-lattice $\mathcal{L}$ being given, to any normalized, nondecreasing, integer-valued, semimodular function $f$ defined on $\mathcal{L}$, we can associate a class of combinatorial geometries called expansions of $f$. The family of expansions of $f$ is shown to have a largest element for the weak map order, $E(f)$, the free expansion of $f$. Expansions generalize and clarify the relationship between two known constructions, one defined by $\mathbf{R}$. P. Dilworth, the other by J. Edmonds and G.-C. Rota.

Further applications are developed for solving two extremal problems of semimodular functions: characterizing

(1) extremal rays of the convex cone of real-valued, nondecreasing, semimodular functions defined on a finite set;

(2) combinatorial geometries which are extremal for the decomposition into a sum.
\end{abstract}

Introduction. $S$ being a finite set, a real-valued function $f$ defined on all subsets of $S$ is said to be semimodular if and only if

$$
f(A)+f(B) \geqslant f(A \cup B)+f(A \cap B), \quad \forall A, B \subset S .
$$

The set of all semimodular, nondecreasing, real-valued functions defined on $S$ forms a convex cone $e_{s}$. Such functions have occurred in a gametheoretical framework [18], and in [4] they are called alternating capacities of order 2. Their importance is especially remarkable in the context of combinatorial geometries, a theorem by Edmonds and Rota [12] stating that each integer-valued, semimodular nondecreasing function $f$ on $S$ defines a unique pregeometry (matroid) $G(f, S)$ called the geometrization of $f$.

This theorem gives the motivation to investigate further connections between semimodular functions and combinatorial geometries, with the objective of developing new constructions of geometries.

The major purpose of this work is to introduce and study a new class of geometric constructions on semimodular nondecreasing, integer-valued, normalized functions. Given any such function $f$ defined on subsets of $S$, we

Received by the editors October 21, 1975 and, in revised form, September 27, 1976.

AMS (MOS) subject classifications (1970). Primary 05B35, 52A40; Secondary 06A20, 15 A39.

${ }^{1}$ This paper contains results from the author's Ph.D. dissertation (Massachusetts Institute of Technology, May 1975) written under the direction of Professor Gian-Carlo Rota. The author wishes to thank Professor Rota for his valuable help and also Professor Thomas Brylawski for many enlightening discussions.

(1) American Mathematical Society 1978 
consider the disjoint union $X$ of the sets $X_{a}$, where for each $a \in S,\left|X_{a}\right|=$ $f(a)$. With the notation that for any subset $A$ of $S, X_{A}=\cup_{a \in A} X_{a}$, one of the main results (Theorem 1.3.3) of this paper is that $\mathcal{H}=\{K \mid K \subset X, \forall A \subset$ $\left.S,\left|K \cap X_{A}\right|>f(A)\right\}$ is the family of dependent sets of a pregeometry on $X$. This theorem guarantees the existence of a class of pregeometries associated to any such function $f$ that we call expansions of $f$.

In $\S 1$, the operation of expansion is defined and some general results are derived. The main property is that for any function $f$ of the cone $\mathcal{C}_{\boldsymbol{s}}$ which is integer-valued and normalized, the operation of expanding $f$ is always possible, and among all expansions of $f$, ordered by the weak map order, there is a largest element, the free expansion of $f, E(f)$. Several characterizations of $E(f)$ are derived, in terms of its circuits, bases and rank-function.

An immediate important application of $E(f)$ is to derive the operation of geometrization as a subgeometry: Edmonds and Rota's theorem becomes a consequence of the existence of the free expansion.

Properties of geometrization are then studied and special consideration is given to the set $\mathcal{S}(G)$ of elements of the cone $\mathcal{C}_{S}$ which are integer-valued, nonnegative and whose geometrization defines a given pregeometry $G$. $\delta(G)$ is characterized and the cases when $\delta(G)$ is a finite set, and more particularly when $\delta(G)$ has exactly two elements, are studied.

$\$ 2$ develops some applications of the idea of expansions to two extremal problems of the following nature. Given a certain decomposition $D$ of elements of the set $\mathcal{C}_{S}$, the question is to characterize the elements of $\mathcal{C}_{S}$ which are $D$-extremal or $D$-irreducible, i.e. which cannot be decomposed into simpler elements of $C_{s}$. The first case we consider is the convex decomposition, i.e. an element $f \in \mathcal{C}_{S}$ is reducible if and only if $\exists a_{1}, a_{2} \in \mathbf{R}$ and $\exists f_{1}, f_{2} \in \mathcal{C}_{S}$, nonproportional to $f$ such that $f=a_{1} f_{1}+a_{2} f_{2}$. The problem is equivalent to characterizing the extremal rays of the cone $\mathcal{C}_{s}$. After proving that a rank-function of a pregeometry is extremal if and only if the pregeometry is connected, we obtain a characterization of extremal integervalued, normalized elements of $\mathcal{C}_{S}$ in terms of expansions (Theorem 2.1.9): such a function is not extremal if and only if some integer multiple of $f$ has a proper disconnected expansion. The second case is a decomposition based on Edmonds' and Rota's theorem, called the sum-decomposition. In this case also the solution is obtained using properties of expansions (Theorem 2.2.1): a pregeometry $G(r, s)$ is sum decomposable if and only if $\mathcal{S}^{+}(G)=\{f \mid f \in$ $\delta(G), f \geqslant 0\}$ contains an element which has a disconnected expansion.

\section{Expansion and geometrization.}

1.1. Basic concepts and notations. This section presents a survey of the basic notions of combinatorial geometries needed in our work. 
A combinatorial pregeometry $G(S)$, or simply a pregeometry $G$, is a set $S$ together with a closure relation $A \rightarrow \bar{A}^{G}$ (or $\bar{A}$ if no ambiguity) for $A, \bar{A} \subset S$, which satisfies the following two axioms:

Exchange Axiom. If $a, b \in S, A \subset S$, and $a \in \overline{A \cup b}-\bar{A}$, then $b \in \overline{A \cup a}$.

FInITE Basis Property. If $A \subset S$, there is a finite subset $A_{0} \subset A$ such that $\overline{A_{0}}=\bar{A}$.

A pregeometry is a geometry if $\varnothing$ and all single-element subsets are closed. The flats of $G(S)$ are the closed subsets of $S$. The set of all flats of $G(S)$ ordered by inclusion is a geometric lattice, i.e. a semimodular point lattice with finite rank.

A subset $A \subset S$ is independent if for no $a \in A, A \subset \overline{A-a}$. If $A$ is not independent, then $A$ is dependent. If $B \subset A \subset S$ and $A \subset \bar{B}$, we say that $B$ spans $A$.

A basis of $A$, for $A \subset S$, is an independent subset of $A$ which spans $A$. All bases of $A$ have the same cardinality, $r(A)$, the rank of $A$. If $A$ is finite, the nullity of $A$ is $n(A)=|A|-r(A)$. The flats of $G(S)$ of rank 1, 2, $r(G)-1$, $r(G)-2$ are called point, line, copoint, coline, respectively.

A circuit is a minimal dependent set. A cyclic flat is a flat which is a union of circuits.

We will use the following cryptomorphic definitions of a pregeometry $G(S)$ :

AXIOMS OF INDEPENDENT SETS. A collection $\mathscr{G}$ of subsets of a set $S$ forms the independent sets of a pregeometry $G(S)$ if and only if:

(1) $I \in \mathcal{G}$ and $J \subset I \Rightarrow J \in \mathcal{G}$;

(2) $A \subset S \Rightarrow$ all maximal subsets of $A$ which are elements of $G$ have the same cardinality.

Axioms of BASES. A collection $\mathscr{B}$ of subsets of a set $S$ is the set of bases of a pregeometry $G(S)$ if and only if:

(1) $\exists n \in \mathbf{N}, n>0$, such that $\forall B \in \mathscr{B},|B|=n$;

(2) if $B_{1}, B_{2} \in \mathscr{B}$ and $x \in B_{1}$, then $\exists y \in B_{2}$ such that $B_{1}-x \cup y \in \mathscr{B}$ (basis exchange property).

AXIOMS OF THE RANK-FUNCTION. An integer-valued function $f$ defined on the subsets of a set $S$ is the rank-function of a pregeometry on $S$ if and only if:

(1) $f(\varnothing)=0, f$ nondecreasing;

(2) $\forall x \in S, 0 \leqslant f(\{x\}) \leqslant 1$;

(3) $\forall A, B \subset S, f(A \cup B)+f(A \cap B) \leqslant f(A)+f(B)$.

A separator of $G$ is a subset $A \subset S$ such that $r(S)=r(A)+r(S-A)$ : every circuit of $G$ is contained either in $A$ or $S-A$ and conversely. If $G$ has no separators other than $\varnothing$ and $S$, then $G$ is connected. $G$ is connected if and only if for any two points $x, y$ of $S$, there is a circuit of $G$ containing both. 
$A \subset S$, the subgeometry of $G$ defined on $A, G-A$, is the pregeometry on $A$ whose closure relation is $U \subset A \rightarrow \bar{U} \cap A$. The contraction of $G$ by $A$, $G / A$, is the pregeometry on $S-A$, with closure $U \subset S-A \rightarrow \overline{U \cup A}-A$. A point $x \in S$ is an isthmus of $G$ if $r(G-x)=r(G)-1 . x$ is a loop of $G$ if $r(G / x)=r(G)$.

Given two geometric lattices $L_{1}$ and $L_{2}$, a strong map from $L_{1}$ to $L_{2}$ is a function $\sigma: L_{1} \rightarrow L_{2}$ which is supremum-preserving and cover-preserving. A strong map $\sigma$ between two pregeometries $G(S)$ and $H(T)$ is a strong map between the corresponding geometric lattices of flats of $G(S)$ and $H(T)$. With the expedient of adjoining a point 0 to each point set $S$ and $T, \sigma$ determines a function $\bar{\sigma}$ from the point set $S \cup 0$ to the point set $T \cup 0$, with $\bar{\sigma}(0)=0 . \bar{\sigma}$ is said to extend to the strong map $\sigma$. A function $\bar{\sigma}$ from $S \cup 0$ to $T \cup 0$ such that $\bar{\sigma}(0)=0$ extends to a strong map from $G(S)$ to $H(T)$ if and only if the inverse image of any flat of $H(T)$ is a flat of $G(S)$.

A function $\bar{\sigma}$ from $S \cup 0$ to $T \cup 0$ extends to a weak map $\sigma$ between $G(S)$ and $H(T)$ if and only if for any set $A \subset S$ such that $\bar{\sigma}(A)$ is independent and $\bar{\sigma}$ is one-one on $A$, then the subset $A$ is independent. The set of all pregeometries defined on a set $S$ is ordered by the weak map order " $G_{1} \leqslant G_{2}$ if and only if the identity function is a weak map from $G_{2}$ to $G_{1}$ "'.

Notations. We will use the following notation throughout the paper. $S$ is a finite set.

$G(r, S)$ is a pregeometry on $S$ with rank-function $r$.

$B(S)$ is the Boolean algebra of subsets of $S$.

$T B_{K}(S)$ is the truncated Boolean algebra of rank $k$ on $S$.

$\mathcal{L}$ stands for a lattice whose least element is denoted by 0 and largest element by 1 . The order defining $\mathcal{L}$ is denoted $\leqslant$ while $A<B$ for $A, B \in \mathcal{L}$ means that $B$ covers $A$. The operations inf and sup are denoted $\wedge$ and $\vee . \mathrm{A}$ subsemilattice $\mathcal{L}^{\prime}$ of $\mathcal{L}$ is a subset of $\mathcal{L}$ which is closed under the operation $\wedge: \forall A, B \in \mathcal{L}^{\prime}, A \wedge B \in \mathcal{L}^{\prime}$.

$\mathcal{C}_{\mathfrak{L}}$ is the set of all real-valued, semimodular, nondecreasing functions defined on $\mathcal{L}$.

$\bigodot_{\mathfrak{L}}^{o}$ is the subset of $\mathcal{C}_{\mathfrak{L}}$ of normalized functions $\left(f \in \mathcal{C}_{\mathfrak{L}}\right.$ is normalized $\Leftrightarrow f(\varnothing)=0$ ).

$I \mathcal{C}_{\mathfrak{L}}$ is the subset of $\mathcal{C}_{\mathfrak{L}}$ of integer-valued functions.

$I \mathcal{C}_{\mathfrak{P}}^{o}$ is the subset of $\mathcal{C}_{\mathfrak{L}}^{o}$ of integer-valued functions.

If $\mathcal{E}$ is the Boolean algebra $B(S)$, we will write $\bigodot_{S}, \bigodot_{S}^{o}, I \bigodot_{S}, I \bigodot_{S}^{o}$ for the corresponding sets.

We will also use the simplified set theoretic notations: $A-a$ for $A \subset S$, $a \in S$ instead of $A-\{a\}$, and $A \cup a$ instead of $A \cup\{a\}$.

1.2. General properties of elements of $\mathcal{C}_{\mathfrak{L}}$. We will consider elements of $\mathcal{C}_{\mathfrak{L}}$, and in the cases that will concern us, $\mathcal{E}$ will be a point lattice: we can use the following local characterization of $\mathcal{C}_{\mathfrak{L}}$ : 
THEOREM 1.2.1. $\mathcal{L}$ being a point lattice, $f \in \mathcal{C}_{\mathfrak{L}}$ if and only if for any $A \in \mathcal{L}$, and any pair of atoms of $\mathcal{L}, a, b$,

$$
f(A \vee a)+f(A \bigvee b) \geqslant f(A)+f(A \vee a \vee b)
$$

Proof. The condition is clearly necessary.

To prove that it is sufficient, we need the following

Lemma 1.2.2. $f \in \mathcal{C}_{\mathcal{P}}$ if and only if $\forall A, B, C \in \mathcal{L}$ with $A \leqslant B$,

$$
f(B \vee C)-f(B) \leqslant f(A \vee C)-f(A) .
$$

Proof. $(\Rightarrow)$ Suppose $A \leqslant B$; we define $A^{\prime}$ to be the complement of $A$ relative to $B$, namely,

$$
A^{\prime}=\bigvee\{a \mid a \in \mathcal{L}, a>0, a \leqslant B, a \leqslant A\} .
$$

By semimodularity,

$$
\begin{aligned}
f\left(A \vee A^{\prime}\right)+f(A \vee C) & \geqslant f\left[\left(A \vee A^{\prime}\right) \wedge(A \vee C)\right]+f\left(A \vee A^{\prime} \vee C\right) \\
& \geqslant f(A)+f(B \vee C) \quad(f \text { nondecreasing })
\end{aligned}
$$

$\Leftrightarrow$ For any $X, Y \in \mathcal{L}$, writing the formula for $A=X \wedge Y, B=Y$, $C=Y$ we get

$$
\begin{aligned}
f(X \vee Y)-f(Y) & \leqslant f[(X \wedge Y) \vee X]-f(X \wedge Y) \\
& =f(X)-f(X \wedge Y) .
\end{aligned}
$$

Thus $f$ is semimodular.

To prove that $f$ is nondecreasing, for any $X, Y \in \mathcal{L}$ such that $X \leqslant Y$, writing the formula with $A=X, B=Y$ and $C=X^{\prime}$, the complement of $X$ relative to $Y$, we get $f(Y)-f(Y) \leqslant f(Y)-f(X)$ or $f(X) \leqslant f(Y)$.

The proof of Lemma 1.2.2 is thus complete and we proceed with Theorem 1.2.1 by proving that the condition is sufficient.

For any $X, Y, Z \in \mathcal{L}$ such that $X \leqslant Y$ we can write $Z=z_{1} \vee z_{2}$ $\vee \cdots \vee z_{p}$ and $Y=X \vee x_{1} \vee \cdots \vee x_{q}$, where the $z_{i}$ 's and $x_{i}^{\prime}$ 's are atoms of $\mathcal{L}$. Then

$$
\begin{aligned}
& f\left(Y \vee z_{1}\right)-f(Y) \\
& \quad=f\left(X \vee x_{1} \vee \cdots \vee x_{q} \vee z_{1}\right)-f\left(X \vee x_{1} \vee \cdots \vee x_{q}\right) \\
& \quad \leqslant f\left(X \vee x_{1} \vee \cdots \vee x_{q-1} \vee z_{1}\right)-f\left(X \vee x_{1} \vee \cdots \vee x_{q-1}\right) \\
& \quad \cdots \\
& \quad \leqslant f\left(X \vee x_{1} \vee z_{1}\right)-f\left(X \vee x_{1}\right) \leqslant f\left(X \vee z_{1}\right)-f(X) .
\end{aligned}
$$

Thus

$$
f\left(Y \vee z_{1}\right)-f(Y) \leqslant f\left(X \vee z_{1}\right)-f(X)
$$

or

$$
f(Y)-f(X) \geqslant f\left(Y \vee z_{1}\right)-f\left(X \vee z_{1}\right)
$$


Repeating the argument with $z_{2}, z_{3}, \ldots, z_{p}$, we get

$$
\begin{aligned}
f(Y)-f(X) & \geqslant f\left(Y \vee z_{1}\right)-f\left(X \vee z_{1}\right) \\
& \geqslant f\left(Y \vee z_{1} \vee z_{2}\right)-f\left(X \vee z_{1} \vee z_{2}\right) \\
& \geqslant \cdots \geqslant f(Y \vee Z)-f(X \vee Z) .
\end{aligned}
$$

Then by Lemma 1.2.2 we know that $f \in \mathcal{C}_{\mathfrak{e}}$.

Theorem 1.2.1 will be especially useful any time we have to construct a semimodular, nondecreasing function: instead of considering the inequalities $f(X)+f(Y) \geqslant f(X \wedge Y)+f(X \vee Y)$ for all elements $X$ and $Y$ of the lattice, we can restrict ourselves to the smaller number of cases when $X$ and $Y$ cover $X \wedge Y$.

$S$ being a finite set, the elements of $e_{S}$ which have attracted most interest are rank-functions, as they define a geometric structure on $S$. In the case of a general element of $e_{s}$, it is interesting to see that the structure induced on $S$, though weaker, bears some analogies with a geometric structure.

For any $f \in \mathcal{C}_{s}$, the operator defined by

$$
A \subset S \rightarrow \bar{A}=\{a \mid a \in S, f(A \cup a)=f(A)\}
$$

is a closure operator, called the $f$-closure. The problem arises of characterizing closure operators which are defined from some element of $f \in \mathcal{C}_{s}$.

Proposition 1.2.3. Any closure operator defined on $S$ is the f-closure for some $f \in \mathcal{C}_{s}$.

Proof. Given a closure operator on $S$ denoted by $A \rightarrow \bar{A}$. The set of closed sets is a subsemilattice $\mathcal{L}$ of $B(S)$ (i.e. $\mathcal{L}$ is closed under set-intersection, and if $A, B \in \mathcal{L}, A \wedge B=\overline{A \cap B}=A \cap B)$.

It is then possible to construct an element $f \in \mathcal{C}_{\mathfrak{R}}$ which is strictly increasing on $\mathcal{L}$ : for example, set $f(S)=0$ and for any element $A$ of $\mathcal{L}$ covered by $S, f(A)=-1 . f$ is then defined inductively by $\forall X \subset S$,

$$
f(X)=\inf \{f(A)+f(B)-f(A \vee B) \mid A>X, B>X\} .
$$

$f$ is semimodular by construction. We have to show that $f$ is strictly increasing. $f$ being defined by induction, and being strictly increasing at the outset, suppose that, until a certain step in the construction, $f$ has been strictly increasing. Let $f(A)$ be the next value to be defined. If $f$ is not strictly increasing, for some $B \in \mathcal{L}, B>A, f(B) \leqslant f(A)$. Let $C$ be any other element of $\mathcal{L}$ covering $A$. By construction

$$
f(A) \leqslant f(B)+f(C)-f(B \vee C)
$$

thus

$$
f(C) \geqslant f(A)-f(B)+f(B \vee C) \geqslant f(B \vee C),
$$


which is a contradiction. So $f$ remains strictly increasing throughout the construction.

Consider the extension of $f$ to all subsets of $S$ defined by $\forall A \subset S$,

$$
f(A)=\inf \{f(L) \mid L \in \mathcal{L}, A \subset L\}=f(\bar{A}) .
$$

To prove that $f \in \mathcal{C}_{S}$, consider $A \subset S$ and $a, b \in S$, and the expression

$$
\begin{aligned}
f(A \cup a) & +f(A \cup b)-f(A)-f(A \cup a \cup b) \\
= & f(\overline{A \cup a})+f(\overline{A \cup b})-f(\bar{A})-f(\overline{A \cup a \cup b}) ;
\end{aligned}
$$

by semimodularity of $f$ on $\mathcal{L}$,

$$
\begin{aligned}
f(\overline{A \cup a})+f(\overline{A \cup b}) & \geqslant f(\overline{A \cup a \cup b})+f[(\overline{A \cup a}) \cap(\overline{A \cup b})] \\
& \geqslant f(\overline{A \cup a \cup b})+f(\bar{A}) .
\end{aligned}
$$

We can then check that the $f$-closure is identical to the given closure: if $A$ is closed, i.e. $A=\bar{A}, \forall a \notin A, f(A \cup a)=f(\overline{A \cup a})>f(\bar{A})=f(A)$ so $A$ is $f$-closed; and if $A$ is f-closed, $\forall a \notin A, f(A \cup a)>f(A)$ so $\bar{A} \neq \overline{A \cup a}$ and $A$ is closed.

Proposition 1.2.3 is thus proved.

The notion of $f$-closure will be handy in the description of some aspects of the two constructions that we will study, expansion and geometrization.

1.3. Expansions. The first geometric construction that we will consider was motivated by the investigation of extremal rays of the cone $e_{s}$. It will be the fundamental construction throughout the paper. The motivation is the following:

Given a pregeometry $G(r, S)$, we consider a partition $P=\left\{P_{1}\right.$, $\left.P_{2}, \ldots, P_{k}\right\}$ of $S$ and define $\mathcal{L}$ as the sublattice of $B(S)$ generated by the subsets $P_{1}, P_{2}, \ldots, P_{k}$. $\mathcal{L}$ is isomorphic to a Boolean algebra. The restriction of $r$ to $\mathcal{L}$ is clearly an element of $I \mathfrak{C}_{\mathfrak{Q}}^{0}$. The question is to find an operation going the opposite way, i.e. given a Boolean algebra $\mathcal{L}$ and an element $f \in I \bigodot_{\mathfrak{Q}}^{o}$, is it possible to find

(1) a set $S$ such that $\mathcal{L}$ is a sublattice of $B(S)$,

(2) a pregeometry on $S$ whose rank-function coincides with $f$ on elements of $\mathcal{L}$ ?

In fact, we will consider a slightly more general setting; $\mathcal{L}$ will be a point lattice and, more precisely, we have

DefinItion 1.3.1. Given a point lattice $\mathcal{L}$, and $f \in I \mathfrak{C}_{\mathfrak{R}}^{\circ}$, a pregeometry $G(r, X)$ (or the rank-function defined on $X$ ) is an expansion of $f$ if and only if:

(i) there is a subsemilattice of $B(X)$ isomorphic to $\mathcal{L}$ (the isomorphism being denoted $\varphi: A \in \mathcal{L} \leftrightarrow \varphi(A) \in B(X)$ );

(ii) $\forall A \in \mathcal{L}, r(\varphi(A))=f(A)$. 
Constructing an expansion of $f$ thus necessitates the construction of a set $X$ and a pregeometry on $X$. We will deal with those problems in turn. For convenience we will also of ten write the image of $A \in \mathcal{E}$ by $\varphi$ as $\varphi(A)=X_{A}$.

If $f(a)=0$ for some atom $a$ of $\mathcal{L}$, the set $X_{a}$ is composed exclusively of loops in any expansion of $f$. If we consider the lattice $\mathcal{L}^{\prime}$ obtained from $\mathcal{L}$ by deleting $a$, and the function $f^{\prime}$ induced on it by $f$, any expansion of $f$ is obtained by adding some loops to an expansion of $f^{\prime}$. Without loss of generality, we will thus assume that for any atom $a$ of $\mathcal{L}, f(a)>0$, and we will consider expansions which have no loop.

Let $\mathcal{Q}$ be the set of atoms of $\mathcal{L} . \forall a, b \in \mathbb{Q}, X_{a} \cap X_{b}=X_{a \wedge b}=X_{0}=\varnothing$, and $\forall a \in \mathbb{Q}, r\left(X_{a}\right)=f(a)$; thus

$$
|X| \geqslant\left|\bigcup_{a \in \mathscr{Q}} X_{a}\right| \geqslant \sum_{a \in \mathscr{Q}} r\left(X_{a}\right)=\sum_{a \in \mathscr{Q}} f(a) .
$$

We will show that, in fact, we can restrict our attention to expansions defined on a set $X$ such that $|X|=\Sigma_{a \in \mathbb{Q}} f(a)$.

Proposition 1.3.2. Given $f \in I \mathcal{C}_{\mathfrak{R}}^{o}$, if $G(r, X)$ is an expansion of $f$, then there is an expansion $G^{\prime}\left(r^{\prime}, X^{\prime}\right)$ of $f$ such that:

(i) $X^{\prime} \subset X$ and $\left|X^{\prime}\right|=\Sigma_{a \in \mathbb{Q}} f(a)$;

(ii) $G^{\prime}$ is a subgeometry of $G$.

PRoof. $\forall a \in \mathbb{Q},\left|X_{a}\right| \geqslant r\left(X_{a}\right)=f(a)$.

For any $a \in \mathcal{Q}$, take a maximal independent set $X_{a}^{\prime}$ of $X_{a}$ and consider the set $X^{\prime}=\cup_{a \in \mathbb{Q}} X_{a}^{\prime}$ : We claim that the subgeometry of $G(r, X)$ defined on $X^{\prime}$ is also an expansion of $f$.

$\forall A \in \mathcal{L}$, to the subset $X_{A}$ we associate the subset $X_{A}^{\prime}=\cup_{a<A} X_{a}^{\prime}$. Clearly the lattice $\left\{X_{A} \mid A \in \mathcal{L}\right\}$ is isomorphic to the lattice $\left\{X_{A}^{\prime} \mid A \in \mathcal{L}\right\}$.

Furthermore, $\forall a \in \mathcal{Q}, r\left(X_{a}^{\prime}\right)=r\left(X_{a}\right)$ so $\forall A \in \mathcal{L}$,

$$
r\left(X_{A}^{\prime}\right)=r\left(\bigcup_{a<A} X_{a}^{\prime}\right)=r\left(\bigcup_{a<A} X_{a}\right)=r\left(X_{A}\right)=f(A) .
$$

In the following, for any $f \in I \mathfrak{C}_{\mathfrak{R}}^{\mathrm{e}}$, we will only consider expansions of $f$ defined on the minimal set $X,|X|=\sum_{a \in \mathbb{Q}} f(a) . X$ is the disjoint union $\cup_{a \in \mathbb{Q}} X_{a}$, where $\left|X_{a}\right|=f(a)$. The isomorphism $\varphi$ is then

$$
A \in \mathcal{L} \rightarrow \varphi(A)=X_{A}=\bigcup_{\substack{a \in \mathbb{Q} \\ a<A}} X_{a} .
$$

One of the main results in this section is that for any $f \in I \mathfrak{C}_{\mathfrak{R}}^{\circ}$, an expansion of $f$ exists.

In any expansion of $f$, if $I \subset X$ is independent, then for any element $A \in \mathcal{E}$, we must have $\left|I \cap X_{A}\right| \leqslant r(A)=f(A)$, in other words, if a set $D$ is dependent there exists an $A \in \mathcal{L}$ such that $\left|D \cap X_{A}\right|>f(A)$. The claim is 
that these conditions are sufficient. More precisely, let us consider the family $\left\{T|T \subset X, \exists A \in \mathcal{L}|, T \cap X_{A} \mid>f(A)\right\}$ and let $\mathcal{K}$ be the set of its minimal elements (for set inclusion).

THEOREM 1.3.3. $\mathcal{H}$ is the set of circuits of an expansion of $f$.

Proof. We will use the following theorem of [8, p. 54]:

"If $\mathcal{H}$ is a family of subsets of a set $S$ no one of which is properly contained in another, $\mathcal{H C}$ is the family of circuits of a pregeometry on $S$ if and only if the following elimination axiom holds:

for any 2 distinct elements $A$ and $B$ of. $\mathcal{I C}$ and any element $x \in A \cap B$ there is an element $C \in \mathcal{H}$ such that $x \notin C$ and $C \subseteq A \cup B$."

We need only show that $\mathcal{H}$ has the elimination axiom.

First, it is easy to see that $\forall T \in \mathscr{H} \Rightarrow \exists A \in \mathcal{L}$ such that $\left|T \cap X_{A}\right|=$ $f(A)+1$.

Consider $T, V \in \mathcal{H}$ (we know then that $\exists A, B \in \mathcal{L}$ such that $\left|T \cap X_{A}\right|=$ $\left.f(A)+1,\left|V \cap X_{B}\right|=f(B)+1\right)$ and $x \in T \cap V$. We want to show that $T \cup V-x=W$ contains an element of $\mathcal{H}$, i.e. $\exists C \in \mathcal{L}$ such that $\mid W \cap$ $X_{C} \mid>f(C)$.

Now, if $\left|W \cap X_{A \wedge B}\right|>f(A \wedge B)$ there is nothing to prove, so suppose that $\left|W \cap X_{A \wedge B}\right| \leqslant f(A \wedge B)$. We have

$$
\begin{aligned}
\left|W \cap X_{A \bigvee B}\right| & \geqslant\left|W \cap\left(X_{A} \cup X_{B}\right)\right|=\left|\left(W \cap X_{A}\right) \cup\left(W \cap X_{B}\right)\right| \\
& =\left|W \cap X_{A}\right|+\left|W \cap X_{B}\right|-\left|W \cap\left(X_{A} \cap X_{B}\right)\right| .
\end{aligned}
$$

Now,

$$
\left|W \cap X_{A}\right|=\left|(T \cup V-x) \cap X_{A}\right| \geqslant\left|T \cap X_{A}\right|-1=f(A) .
$$

Similarly, $\left|W \cap X_{B}\right| \geqslant f(B)$. On the other hand,

$$
\left|W \cap\left(X_{A} \cap X_{B}\right)\right|=\left|W \cap X_{A \wedge B}\right| \leqslant f(A \wedge B) .
$$

Finally,

$$
\left|W \cap X_{A \bigvee B}\right| \geqslant f(A)+f(B)-f(A \wedge B) \geqslant f(A \vee B)
$$

If $\left|W \cap X_{A \vee B}\right|=f(A \vee B)$, the following equalities hold:

(a) $\left|W \cap X_{A}\right|=\left|(T \cup V-x) \cap X_{A}\right|=\left|T \cap X_{A}\right|-1$,

(b) $\left|W \cap X_{B}\right|=\left|(T \cap V-x) \cap X_{B}\right|=\left|T \cap X_{B}\right|-1$,

(c) $\left|W \cap X_{A \wedge B}\right|=f(A \wedge B)$.

(a) implies $x \in X_{A}$ and $V \cap X_{A}=T \cap V \cap X_{A}$, and similarly, from (b), $x \in X_{B}$ and $T \cap X_{B}=T \cap V \cap X_{B}$.

We have

$$
\begin{aligned}
\left|(T \cup V) \cap X_{A \wedge B}\right| & =\left|(T \cup V-x) \cap X_{A \wedge B}\right|+1=\left|W \cap X_{A \wedge B}\right|+1 \\
& =f(A \wedge B)+1 .
\end{aligned}
$$


On the other hand,

and

$$
\begin{aligned}
(T \cup V) \cap X_{A \wedge B} & =\left(T \cap X_{A} \cap X_{B}\right) \cup\left(V \cap X_{A} \cap X_{B}\right) \\
& =T \cap V \cap X_{A} \cap X_{B}
\end{aligned}
$$

because $T \cap V \notin \mathcal{H}$.

$$
\left|(T \cup V) \cap X_{A \wedge B}\right|=\left|(T \cap V) \cap X_{A \wedge B}\right| \leqslant f(A \wedge B)
$$

The hypothesis $\left|W \cap X_{A \vee B}\right|=f(A \vee B)$ being rejected, we have found an element of $\mathcal{L}, A \vee B$, such that $\left|W \cap X_{A \vee B}\right|>f(A \vee B)$. $\mathcal{H}$ is thus the set of circuits of a pregeometry $G(\rho, S)$. The family of dependent sets is $\left\{D|D \subset X, \exists A \in \mathcal{L}|, D \cap X_{A} \mid>f(A)\right\}$, the family of independent sets is $\left\{I|I \subset X, \forall A \in \mathcal{L}|, I \cap X_{A} \mid \leqslant f(A)\right\}$. It remains to show that $\forall A \in \mathcal{L}$, $\rho(A)=f(A)$ (clearly we have $\rho(A) \leqslant f(A)$ ).

LEMMA 1.3.4. $\forall T \subset X, \rho(T) \geqslant \inf _{A \in \mathfrak{Q}}\left\{f(A)+\left|T-X_{A}\right|\right\}$.

Proof. Let $I$ be a maximal independent set contained in $T: T=I \cup x_{1} \cup$ $x_{2} \cup \cdots \cup x_{k}$ where $x_{i} \notin I . \forall i, 1 \leqslant i \leqslant k, I \cup x_{i}$ is dependent: $\exists A_{i} \in \mathcal{L}$ such that $\left|\left(I \cup x_{i}\right) \cap X_{A_{i}}\right|>f\left(A_{i}\right)$. I being independent, we also have $\mid I \cap$ $X_{A_{i}} \mid \leqslant f\left(A_{i}\right)$; thus $\left|I \cap X_{A_{i}}\right|=f\left(A_{i}\right)$ and $x_{i} \in X_{A_{i}}$.

Using the fact that for any $A, B \in \mathcal{L}$, such that $\left|I \cap X_{A}\right|=f(A)$ and $\left|I \cap X_{B}\right|=f(B)$, we have $\left|I \cap\left(X_{A} \cup X_{B}\right)\right|=\left|I \cap X_{A \vee B}\right|=f(A \vee B)$ (proof:

we can infer that

$$
\begin{aligned}
f(A \vee B) & \geqslant\left|I \cap X_{A \vee B}\right| \geqslant\left|I \cap\left(X_{A} \cup X_{B}\right)\right| \\
& =\left|I \cap X_{A}\right|+\left|I \cap X_{B}\right|-\left|I \cap X_{A} \cap X_{B}\right| \\
& \geqslant f(A)+f(B)-f(A \wedge B) \geqslant f(A \vee B) .),
\end{aligned}
$$

$$
\left|I \cap\left(\bigcup_{i=1}^{k} X_{A_{i}}\right)\right|=\left|I \cap X_{\bigvee_{i=1}^{k} A_{i}}\right|=f\left(\bigvee_{i=1}^{k} A_{i}\right)
$$

Then

$$
\begin{aligned}
f\left(\bigvee_{i=1}^{k} A_{i}\right)+\mid T- & \bigcup_{i=1}^{k} X_{A_{i}} \mid \\
& =f\left(\bigvee_{i=1}^{k} A_{i}\right)+\left|\left(I \cup x_{1} \cup x_{2} \cup \cdots \cup x_{k}\right)-\bigcup_{i=1}^{k} X_{A_{i}}\right| \\
& \left.=f\left(\bigvee_{i=1}^{k} A_{i}\right)+\left|I-\bigcup_{i=1}^{k} X_{A_{i}}\right| \quad \text { (because } x_{i} \in X_{A_{i}}\right) \\
& =\left|I \cap \bigcup_{i=1}^{k} X_{A_{i}}\right|+\left|I-\bigcup_{i=1}^{k} X_{A_{i}}\right|=|I|=\rho(T) .
\end{aligned}
$$


So we have $\rho(T) \geqslant \inf _{A \in \mathbb{R}}\left\{f(A)+\left|T-X_{A}\right|\right\}$.

It follows from Lemma 1.3.4 that $\forall A \in \mathcal{L}$,

$$
\rho\left(X_{A}\right) \geqslant \inf _{B \in \mathcal{L}}\left\{f(B)+\left|X_{A}-X_{B}\right|\right\} \geqslant f(A)
$$

and, thus, $\rho(A)=f(A)$. The pregeometry $G(\rho, S)$ is an expansion of $f$.

Corollary. 1.3.5. If $f(1)=M$, the family of bases of $G(\rho, S)$ is

$$
\mathscr{B}=\left\{B|B \subset X,| B|=M, \forall A \in \mathcal{L},| B \cap X_{A} \mid \leqslant f(A)\right\} .
$$

Theorem 1.3.3 is a special version of Dilworth's embedding of a lattice into a geometric lattice [5], and a slight modification of Dilworth's proof can be a (somewhat longer) alternative for proving it [5, pp. 125-131].

Given an element $f \in I \mathcal{C}_{\mathfrak{R}}^{o}$, the set of expansions of $f$ is, thus, never empty. If we restrict our attention to expansions defined on the minimal cardinality set $X,|X|=\sum_{a \in \mathbb{Q}} f(a)$, the set of all those expansions, $\mathscr{E}(f, X)$, which is a subset of the set of all pregeometries defined on $X$, is endowed with the induced weak map order.

THEOREM 1.3.6. $\mathcal{E}(f, X)$, ordered by the weak map order, has a largest element.

Proof. Theorem 1.3.6 is a consequence of Corollary 1.3.5: let the pregeometry on $X$ defined by $\mathscr{B}$ be denoted by $E(f, X)$.

If $G$ is an element of $\mathcal{E}(f, X)$, any basis $B$ of $G$ must verify the conditions: $|B|=M$ and $\forall A \in \mathcal{L},|B \cap \varphi(A)| \leqslant f(A)$ so that $B \in \mathscr{B}: G$ is thus a weak map image of $E(f, X)$, and $E(f, X)$ is the largest element of $\mathcal{E}(f, X)$.

$E(f, X)$ will be called the free expansion of $f$. We will also write $E(f)$ when there is no ambiguity, and $r_{E}$ will be its rank-function. As before, we will write $\varphi(A)=X_{A}$.

Proposition 1.3.7. A set $X_{A}$ is independent in $E(f)$ if and only if $f(A)=$ $\Sigma_{a \in \mathbb{Q} ; a \leqslant A} f(a)$.

Proof. Suppose $X_{A}$ is independent. Then $\forall B \in \mathcal{L},\left|X_{A} \cap X_{B}\right| \leqslant f(B)$, in particular, for $B=A$,

$$
\sum_{\substack{a \in \mathbb{A} \\ a<A}} f(a)=\sum_{\substack{a \in \mathbb{A} \\ a<A}}\left|X_{a}\right|=\left|X_{A}\right| \leqslant f(A) .
$$

By semimodularity we must have $f(A)=\sum_{a \in \mathbb{Q} ; a \leqslant A} f(a)$.

Conversely, if $f(A)=\Sigma_{a \in \mathbb{Q} ; a \leqslant A} f(a)$, then for any subset $A^{\prime} \subset A$, we also have

$$
f\left(A^{\prime}\right)=\sum_{\substack{a \in \mathbb{Q} \\ a<A^{\prime}}} f(a)
$$


$\forall B \in \mathcal{L}$,

$$
\left|X_{A} \cap X_{B}\right|=\left|X_{A \wedge B}\right|=\sum_{\substack{a \in \mathcal{Q} \\ a<A \wedge B}} f(a)=f(A \wedge B) \leqslant f(B),
$$

so $X_{A}$ is independent in $E(f)$. e.

Proposition 1.3.8. A set $X_{A}$ is closed in $E(f)$ if and only if $A$ is f-closed in

Proof. If $A$ is not $f$-closed in $\mathcal{L}$, for some $B \in \mathcal{L}, A \leqslant B$ and $A \neq B$, we have $f(A)=f(B), X_{A} \subset X_{B}$ and $r_{E}\left(X_{A}\right)=r_{E}\left(X_{B}\right)$ with $X_{A} \neq X_{B}: X_{A}$ is not closed in $E(f)$.

Suppose now that $A$ is $f$-closed in $\mathcal{L}: \forall a \in \mathbb{Q}, a \leqslant A, f(A \vee a)>f(A)$. (This also implies that $\forall B \leqslant A, f(B \vee a)>f(B)$.) To show that $X_{A}$ is closed, we will show that given any maximal independent set $I \subset X_{A}$, for any $x \in X, x \notin X_{A}, I \cup x$ is an independent set.

$x \in X \Rightarrow$ for some $a \in \mathbb{Q}, x \in X_{a}(a \$ A)$;

$\forall B \in \mathcal{L}$, we have: if $a \nless B$,

$$
\left|(I \cup x) \cap X_{B}\right|=\left|I \cap X_{B}\right| \leqslant f(B) ;
$$

if $a \leqslant B$,

$$
\begin{aligned}
\left|(I \cup x) \cap X_{B}\right| & =\left|I \cap X_{B}\right|+1 \leqslant r_{E}\left(X_{A} \cap X_{B}\right)+1 \\
& =f(A \wedge B)+1 \leqslant f[(A \wedge B) \vee a] \leqslant f(B) .
\end{aligned}
$$

Proposition 1.3.9. The rank-function of $E(f), r_{E}$, is given by

$$
\forall T \subset X, \quad r_{E}(T)=\inf _{A \in \mathcal{L}}\left\{f(A)+\left|T-X_{A}\right|\right\} .
$$

Proof. Given $T \subset X$, let $I$ be an independent set contained in $T$. For any $A \in \mathcal{L}$,

$$
|I|=\left|I \cap X_{A}\right|+\left|I-X_{A}\right| \leqslant f(A)+\left|I-X_{A}\right| \leqslant f(A)+\left|T-X_{A}\right| .
$$

In particular, for a maximal independent set of $T$, we have

$$
r_{E}(T) \leqslant f(A)+\left|T-X_{A}\right|, \quad \forall A \in \mathcal{L} .
$$

On the other hand, by Lemma 1.3.4,

$$
r_{E}(T) \geqslant \inf _{A \in \mathcal{L}}\left\{f(A)+\left|T-X_{A}\right|\right\} .
$$

Another way to associate a pregeometry on $S$ to any element of $I \bigodot_{S}^{o}$ has been investigated by Edmonds and Rota [12]: the first important application of the notion of expansion will be to derive Edmonds' and Rota's theorem.

TheOREM 1.3.10 (EDMONDS-Rota). Given an element $f \in I \mathrm{C}_{S}$,

(i) the subsets $A \subset S$ such that, $\forall A^{\prime} \subset A, A^{\prime} \neq \varnothing,\left|A^{\prime}\right| \leqslant f\left(A^{\prime}\right)$, are the independent sets of a pregeometry on $S$, called the geometrization $G(f, S)$ of $f$; 
(ii) if $f$ is nonnegative, the rank-function $r$ of $G(f, S)$ is given by:

$$
\forall T \subset S, \quad T \neq \varnothing, \quad r(T)=\inf _{A \subset T}\{f(A)+|T-A|\} .
$$

Given an element $f \in I \bigodot_{S}^{o}$, let $E(f, X)$ be its free expansion. To each point $a \in S$ corresponds the subset $\varphi(a) \subset X$ such that $f(a)=|\varphi(a)|$ (as before, we suppose that $\forall a \in S, f(a)>0$; the general case will be considered later). In each subset $\varphi(a)$, for $a \in S$, we choose arbitrarily a point $p_{a}$, and $\forall A \subset S$, we call $p_{A}=\left\{p_{a} \mid a \in A\right\}$. Let $G\left(f, p_{S}\right)$ be the subgeometry of $E(f, X)$ defined on $p_{S}$. i.e.

A set $p_{A} \subset p_{S}$ is independent in $G\left(f, p_{S}\right)$ iff it is independent in $E(f, X)$,

$$
\forall T \subset S, \quad\left|p_{A} \cap \varphi(T)\right| \leqslant f(T)
$$

but

$$
\left|p_{A} \cap \varphi(T)\right|=\left|p_{A} \cap\left(\bigcup_{a \in T} \varphi(a)\right)\right|=|A \cap T| .
$$

Thus the condition is equivalent to

$p_{A}$ is independent in $G\left(f, p_{S}\right)$ if and only if $\forall A^{\prime} \subset A,\left|A^{\prime}\right| \leqslant f\left(A^{\prime}\right)$.

If we now consider the family $\mathcal{G}$ of subsets of $S$ defined by

$$
\mathcal{G}=\left\{A\left|A \subset S, \forall A^{\prime} \subset A,\right| A^{\prime} \mid \leqslant f\left(A^{\prime}\right)\right\},
$$

because of the bijection $p_{A} \rightarrow A$ from $p_{S}$ to $S, \emptyset$ is the family of independent sets of a pregeometry defined on $S$, isomorphic to $G\left(f, p_{S}\right)$ : denoted by $G(f, S)$, it is the geometrization of $f$.

The rank-function of $G(f, S)$ is given by (Proposition 1.3.9), $\forall T \subset S$,

$$
\begin{aligned}
r(T) & =r\left(p_{T}\right)=\inf _{A \subset S}\left\{f(A)+\left|p_{T}-(A)\right|\right\} \\
& =\inf _{A \subset S}\{f(A)+|T-A|\} \\
& =\inf _{A \subset T}\{f(A)+|T-A|\} .
\end{aligned}
$$

Till now, we have supposed that $f$ is normalized and positive on points of $S$. In the general case when $f \in I \mathcal{C}_{S}$, we can show that the family $\mathscr{G} \cup\{\varnothing\}$, where

$$
\mathcal{G}=\left\{A\left|A \subset S, \forall A^{\prime} \subset A, A^{\prime} \neq \varnothing,\right| A^{\prime} \mid \leqslant f\left(A^{\prime}\right)\right\},
$$

is the family of independent sets of a pregeometry on $S$.

(1) Let $S^{\prime} \subset S$ be defined by $S^{\prime}=\{x \mid x \in S, f(x)>0\}$. Set a function $f^{\prime}$ defined on $S^{\prime}$ as $f^{\prime}(\varnothing)=0$ and $\forall A \subset S^{\prime}, A \neq \varnothing, f^{\prime}(A)=f(A)$. $f^{\prime}$ defines a pregeometry $G\left(f^{\prime}, S^{\prime}\right)$ on $S^{\prime}$ as described above.

(2) Consider the pregeometry $G(f, S)$ on $S$ defined as the addition to $G\left(f^{\prime}, S^{\prime}\right)$ of a set of loops $S-S^{\prime}$. The independent sets of $G(f, S)$ are 
exactly the elements of $G \cup\{\varnothing\}$ : clearly any subset $A$ such that $A \cap(S-$ $\left.S^{\prime}\right) \neq \varnothing$ does not belong to $\mathscr{G} \cup\{\varnothing\}$; and if $A \cap\left(S-S^{\prime}\right)=\varnothing, A \in \mathscr{G}$ if and only if $A$ is independent in $G\left(f^{\prime}, S^{\prime}\right)$.

We have thus proved Edmonds' and Rota's theorem in its generality.

A slightly more general formulation of the theorem is the following:

Corollary 1.3.11. Given $\mathcal{L}$, a subsemilattice of $B(S)$ containing $S$ and $\varnothing$, any element $f$ of $I \mathcal{C}_{\mathfrak{L}}$ defines a pregeometry on $S$, whose independent sets are

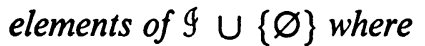

$$
\mathcal{G}=\{I|I \subset S, \forall A \in \mathcal{L}, A \neq \varnothing,| I \cap A \mid \leqslant f(A)\} .
$$

As a result of our preceding proof we have the following theorem which makes explicit the relationship between expansion and geometrization.

THEOREM 1.3.12. Given an element $f$ of $I \bigodot_{S}^{o}$ the geometrization of $f$ is a subgeometry of its free expansion.

Notation. While $G(f, S)$ means the geometrization of some element $f \in$ $I C_{S}$, when we use the letter $r, G(r, S)$ will mean that $r$ is effectively the rank-function of $G(r, S)$.

Before presenting some other applications of the concept of expansion, we will first study some properties of geometrizations which will be useful in the sequence.

1.4. Geometrization. We will often study the geometrization $G(f, S)$ of an element $f \in I \bigodot_{S}$ by means of its circuits.

Proposition 1.4.1. Given $f \in I \bigodot_{S}$, a subset $K \subset S,|K| \geqslant 2$ is a circuit of $G(f, S)$ if and only if $|K|=f(K)+1$ and $\forall K^{\prime} \subsetneq K, f\left(K^{\prime}\right) \geqslant\left|K^{\prime}\right|$.

Proof. The condition means that $K$ is dependent and any subset $K^{\prime}$ of $K$ is independent. Thus $K$ is a circuit of $G(f, S)$.

Conversely, let $K$ be a circuit of $G(f, S)$. $K$ is dependent $\Rightarrow \exists K_{0} \subset K$ such that $f\left(K_{0}\right)<\left|K_{0}\right|\left(K_{0} \neq \varnothing\right)$, but for any subset $K^{\prime} \subset K, K^{\prime} \neq K, K^{\prime}$ is independent and, thus, $\left|K^{\prime}\right| \leqslant f\left(K^{\prime}\right)\left(K^{\prime} \neq \varnothing\right)$. Thus $K_{0}=K$ and $f(K)<$ $|K|$.

On the other hand, $\forall x \in K$,

$$
f(K) \geqslant f(K-x) \geqslant|K-x|=|K|-1 \geqslant f(K) .
$$

We have equality all along and, thus, $f(K)=|K|-1$.

We have to exclude the case $|K|=1$, i.e. $K$ is a loop because the condition of Proposition 1.4.1 is not necessary then.

ExAmple. $S=\{a, b\} . f$ is defined by $f(\varnothing)=-4, f(a)=1, f(b)=-3$, $f(a b)=1 . b$ is a loop and no relation of the above type exists.

A set $A \subset S$ is said to be normal in $G(f, S)$ iff $A$ is independent in $G(f, S)$ 
and $f(A)=|A|$. From the proof of Proposition 1.4.1, we see that if $K$ is a circuit of $G(f, S)$ and $|K| \geqslant 2$, then for any $x \in K, K-x$ is a normal set in $G(f, S)$.

Proposition 1.4.2. If a point $a \in S$ is contained in no normal subset of $G(f, S)$, then a is either a loop or an isthmus of $G(f, S)$.

Proof. Suppose $a$ is neither a loop nor an isthmus of $G(f, s)$ : there is a circuit $K$ such that $a \in K$, and $|K| \geqslant 2: \forall x \in K, x \neq a, K-x$ is normal and contains $a$, which is a contradiction.

The closure operator associated to $G(f, S)$ is described by the following theorem:

THEOREM 1.4.3. Given $f \in I \varrho_{S}$, let $U$ be the set of all loops and isthmuses of $G(f, S)$.

(i) If $A$ is an independent set of $G(f, S), A-U$ is partitioned by its maximal normal subsets.

(ii) An element $a \in S, a \notin U$, is in the closure $\bar{A}$ of a subset $A \subset S$ if and only if there is a normal subset $B \subset A$ such that $f(B)=f(B \cup a)$.

(iii) If $A$ is a flat of $G(f, S), A-U$. is partitioned by its maximal $f$-closed subsets.

Proof. All the above results are generalizations of results of [8, Chapter 7]; the same proofs can be used here, and we will not repeat them.

We have seen that any element $f \in I \mathcal{E}_{s}$ defines a unique pregeometry $G(f, S)$, but, conversely, to a given pregeometry $G(r, S)$ there may be many elements of $I e_{S}$ whose geometrization is $G(r, S)$.

Given a pregeometry $G(r, S)$, let $\S(G)$ be the subset of $I \mathcal{C}_{S}$ defined by

$$
\varsigma(G)=\left\{f \mid f \in I C_{s}, G(f, S)=G(r, S)\right\} .
$$

The set $\delta(G)$ is characterized in the following way.

THEOREM 1.4.4. Given a pregeometry $G(r, S)$, an element $f \in I \bigodot_{S}$ belongs to $\mathcal{S}(G)$ if and only if:

(i) $f(A) \geqslant r(A)$ for any $A \subset S, A$ not consisting exclusively of loops of $G$;

(ii) $f(a) \leqslant 0$ for any loop a of $G$;

(iii) $f(K)=r(K)$ for any circuit $K$ of $G,|K| \geqslant 2$.

Proof. Let $f \in \delta(G)$. Let $A \subset S, A \neq \varnothing$ : if $A$ is independent, $\forall A^{\prime} \subset A$, $A^{\prime} \neq \varnothing, f\left(A^{\prime}\right) \geqslant\left|A^{\prime}\right|$, in particular, for $A^{\prime}=A, f(A) \geqslant|A|=r(A)$.

If $A$ is dependent, and if $A$ is not a set of loops of $G, A$ contains a maximal independent set $I_{A}, I_{A} \neq \varnothing$ :

$$
r(A)=r\left(I_{A}\right) \leqslant f\left(I_{A}\right) \leqslant f(A) .
$$

Thus (i) is proved to be necessary. 
If $a$ is a loop of $G$, clearly, $f(a) \leqslant 0$ (otherwise, $a$ would be independent in $G)$.

Finally, by Proposition 1.4.1, if $K$ is a circuit of $G,|K| \geqslant 2, f(K)=|K|-$ $1=r(K)$. The necessary part of 1.4 .4 is proved.

Suppose now that an element $f \in I \mathcal{C}_{S}$ verifies (i), (ii) and (iii). First, by (ii) any loop of $G$ is a loop of $G(f, S)$.

Let $A$ be an independent set of $G: \forall A^{\prime} \subset A, A^{\prime}$ is independent in $G$, by (i) we have $f\left(A^{\prime}\right) \geqslant r\left(A^{\prime}\right)=\left|A^{\prime}\right| . A$ is thus independent in $G(f, S)$.

Let $A$ be dependent in $G$. $A$ contains a circuit $K$ of $G$.

If $|K|=1, K$ is a loop of $G$, and a loop of $G(f, S)$, so $A$ is dependent in $G(f, S)$.

If $|K| \geqslant 2$, then $f(K)=r(K)=|K|-1: K$ is dependent in $G(f, S)$, so is A.

Thus $G$ and $G(f, S)$ have the same independent sets and they are identical.

When we are only interested in nonnegative elements of $I C_{S}$, we define $\mathcal{S}^{+}(G)$ as being

$$
\mathcal{S}^{+}(G)=\{f \mid f \in \mathcal{S}(G), f \geqslant 0\} .
$$

COROLlaRY 1.4.5. Given a pregeometry $G(r, S), f \in I \mathcal{C}_{S}$ belongs to $\mathcal{S}^{+}(G)$ if and only if:

(i) $f(A)>r(A), \forall A \subset S$;

(ii) $f(K)=r(K)$ for any circuit $K$ of $G$.

We say that $\mathcal{S}^{+}(G)$ is bounded if there is an integer $M \geqslant 0$ such that $\forall f \in \mathcal{S}^{+}(G), f<M$. Clearly, $\mathcal{S}^{+}(G)$ has a finite number of elements if and only if $\mathcal{S}^{+}(G)$ is bounded.

Proposition 1.4.6. Given a pregeometry $G(r, S), \varsigma^{+}(G)$ has a finite number of elements if and only if $G$ has no isthmus.

Proof. If $G$ has no isthmus, $\cup_{K \text { circuit of } G} K=S . \forall f \in \mathcal{S}^{+}(G)$ and $\forall A \subset S$,

$$
f(A) \leqslant f(S) \leqslant \sum_{K \text { circuit of } G} f(K)=\sum_{K \text { circuit of } G}(|K|-1) .
$$

$\mathcal{S}^{+}(G)$ is thus bounded and has a finite number of elements. by

Conversely, suppose $G$ has an isthmus $a$. Consider the function $\delta_{a}$ defined

$$
\begin{aligned}
\delta_{a}(A) & =1 \quad \text { if } a \in A, \quad \forall A \subset S . \\
& =0 \quad \text { if } a \notin A,
\end{aligned}
$$

Clearly $\delta_{a} \in I \bigodot_{S}^{o}$, and $\forall k \in N$ we want to show that $r+k \delta_{a} \in \mathcal{S}^{+}(G)$. 
$\forall A \subset S$, we have $\left(r+k \delta_{a}\right)(A) \geqslant r(A)$, and if $K$ is a circuit of $G$, necessarily $a \notin K$, and $\left(r+k \delta_{a}\right)(K)=r(K)$. By Corollary 1.4.5, $r+k \delta_{a} \in$ $\mathcal{S}^{+}(G)$, and $\mathcal{S}^{+}(G)$ has an infinite number of elements.

Among the cases when $\mathcal{S}^{+}(G)$ has a finite number of elements, we can investigate the cases when $\left|\delta^{+}(G)\right|$ is minimal. Supposing that $G(r, S)$ is loopless, we always have $\left|\mathcal{S}^{+}(G)\right|>1$ : take the function $f$ defined by:

$$
\forall A \subset S, f(A)=r(A) \text { if } A \neq \varnothing \text {, and } f(\varnothing)=1 \text {. }
$$

Clearly $f \in \mathcal{S}^{+}(G)$ and $f>r: f$ and $r$ are two distinct elements of $\mathcal{S}^{+}(G)$. In the following we will characterize pregeometries such that $\left|\delta^{+}(G)\right|=2$.

LEMMA 1.4.7. $G$ being a loopless pregeometry, if $\left|\delta^{+}(G)\right|>2$, there is an element $f \in \mathcal{S}^{+}(G)$ such that $\exists p \in S, f(p)=2$ and $\exists a \in S, a \neq p, f(a)=1$.

Proof. Suppose $\left|\mathcal{I}^{+}(G)\right|>2$ and let $f$ be an element of $\mathcal{S}^{+}(G)$ such that $f$ is normalized and $f \neq r$.

Let $E(f, X)$ be the free expansion of $f$, and $r_{E}$ its rank-function. Using the notation used in the proof of Theorem 1.3.10, we know that by arbitrarily taking a point $p_{a}$ in each $\varphi(a)$ for $a \in S$, the subgeometry of $E(f, X)$ defined on $p_{S}=\left\{p_{a} \mid a \in S\right\}$ is isomorphic to the geometrization of $f$, in this case to $G$ itself as $f \in \mathcal{S}^{+}(G): \forall A \subset S, r_{E}\left(p_{A}\right)=r(A)$.

Let $u \in S$ be a point such that $f(u)>1$ (such a point exists: if not, $f \equiv r$ ), $|\varphi(u)|>1$. Let $y$ be a point of $\varphi(u)$ such that $y \neq p_{u}$. Let $B$ be the Boolean algebra sublattice of $B(X)$ generated by the subsets $\left\{p_{a}\right\}, a \in S, a \neq u$ and $\left\{p_{u}, y\right\} . B$ is isomorphic to $B(S)$. The restriction of $r_{E}$ to $B$ is a function $g$ which we can consider as being defined on $B(S)$ : we have

$$
g(u)=g\left(\left\{p_{u}, y\right\}\right)=r_{E}\left(\left\{p_{u}, y\right\}\right)=2,
$$

$\forall a \in S, a \neq u$,

$$
g(a)=g\left(\left\{p_{a}\right\}\right)=r_{E}\left(\left\{p_{a}\right\}\right)=1 .
$$

We claim that $g \in \mathcal{S}^{+}(G) . \forall A \subset S$, if $u \notin A$,

$$
g(A)=g\left(p_{A}\right)=r_{E}\left(p_{A}\right)=r(A)
$$

if $u \in A$,

$$
\begin{aligned}
g(A) & =g\left(\left\{p_{u}, y\right\} \cup p_{A-u}\right)=r_{E}\left(p_{A-u} \cup p_{u} \cup y\right) \\
& =r_{E}\left(p_{A-u} \cup p_{u}\right)=r_{E}\left(p_{A}\right)=r(A),
\end{aligned}
$$

so $\forall A \subset S, \mathbf{g}(A) \geqslant r(A)$.

Let $K$ be a circuit of $G$ : if $u \notin K$, as for above, $g(K)=r(K)$; suppose $p \in K: g(K)=r_{E}\left(p_{K-u} \cup x_{u} \cup y\right)$. Using the properties of the free expansion $E(f, X)$, we have

$$
f(K)=r_{E}(\varphi(K)) \geqslant r_{E}\left(p_{K-u} \cup p_{u} \cup y\right) \geqslant r_{E}\left(p_{K}\right)=r(K),
$$


but $f \in \mathcal{S}^{+}(G) \Rightarrow f(K)=r(K)$, so we have equality all along: $g(K)=$ $r_{E}\left(p_{K-u} \cup p_{u} \cup y\right)=r(K)$. So $g \in \mathcal{S}^{+}(G)$.

THEOREM 1.4.8. $G$ being a loopless pregeometry, $\left|\varsigma^{+}(G)\right|=2$ if and only if $\forall a \in S, \bar{a}$ (closure of a) belongs to the modular cut generated by the closures of all the circuits of $G$ containing $a$.

Proof. Suppose $\left|\delta^{+}(G)\right|>2$; by Lemma 1.4.7, $\exists f \in \delta^{+}(G)$ such that $\exists u \in S, f(u)=2$ and $\forall a \in S, a \neq u, f(a)=1$. Let $E(f, X)$ be the free expansion of $f .|X|=|S|+1$; if $\varphi(u)=\{u, v\}$, we know that the subgeometry of $E(f, X)$ defined on $X-\{v\}$ is isomorphic to $G$, thus $E(f, X)$ can be considered as a single-point extension of $G . \forall K$ circuit of $G$, containing $u$,

$$
r_{E}(K)=r(K)=f(K)=r_{E}(\varphi(K))=r_{E}(K \cup v),
$$

so $v \in \bar{K}^{E}$. Thus $\bar{K}^{G}$ is in the modular cut $M$ defining the extension by $v$.

Furthermore, $\forall K$ circuit of $G$ containing $u,\{u, v\} \subset \bar{K}^{E}$ and $r_{E}(\{u, v\})>$ 1: $\bar{u}^{G}$ does not belong to $M$.

Conversely, suppose that there is a point $u \in S$, such that $\bar{u}$ does not belong to the modular cut $M$ generated by all the circuits of $G$ containing $u$. Consider the single-element extension defined by $M: H(X)$, where $X=S \cup$ $v$. Define a function $f$ on $B(S)$ as follows:

$$
\begin{aligned}
& \text { if } u \notin A, \quad f(A)=r_{H}(A), \\
& \text { if } u \in A, \quad f(A)=r_{H}(A \cup v), \quad \forall A \subset S .
\end{aligned}
$$

We have $f(u)=2$ and $\forall a \in S, a \neq u, f(a)=1$. We claim that $f \in \mathcal{S}^{+}(G)$.

If $u \notin A, \quad f(A)=r_{H}(A)=r(A)$,

$$
\text { if } u \in A, \quad f(A)=r_{H}(A \cup v) \geqslant r_{H}(A)=r(A), \quad \forall A \subset S .
$$

$\forall$ circuit $K$ of $G$,

$$
\begin{array}{ll}
\text { if } u \notin K, & f(K)=r_{H}(K)=r(K), \\
\text { if } u \in K, & f(K)=r_{H}(K \cup v)=r_{H}(K)=r(K)
\end{array}
$$

(because $\bar{K}^{G}$ is in $M$ and $\overline{K-v^{H}}=\bar{K}^{H}$ ). Finally, $f \in \mathcal{S}^{+}(G)$ with $f>r$ : $\left|\delta^{+}(G)\right|>2$. Theorem 1.4.8 is thus proved.

COROLlary 1.4.9. A necessary condition for having $\left|\mathcal{S}^{+}(G)\right|=2$ is that $\forall a \in S, r\left(\cap\left\{\bar{K}^{G} \mid K\right.\right.$ circuit of $\left.\left.G, a \in K\right\}\right)=1$.

As illustrations for Theorem 1.4.8, we can say that $\left|\varsigma^{+}(G)\right|=2$ for the following $G$ 's: 


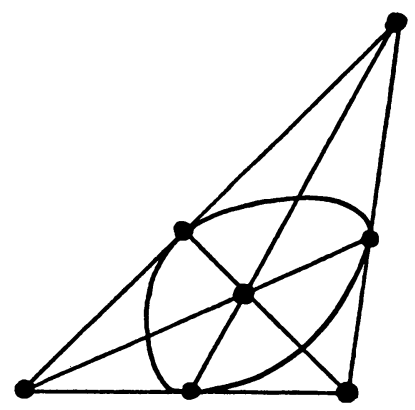

FANO PLANE

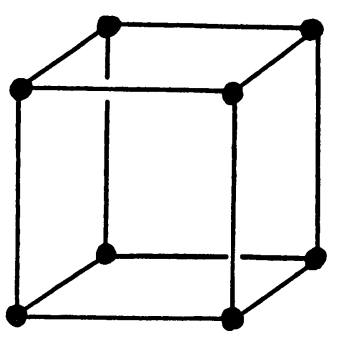

CUBE IN 3-SPACE

$\delta^{+}(G)$ is an ordered set, whose order relation is induced by the order of $\mathbf{R}^{|B(S)|}$. In general, $\mathcal{S}^{+}(G)$, when finite, has no unique maximal element. For example, let $G$ be the geometry on 5 points:

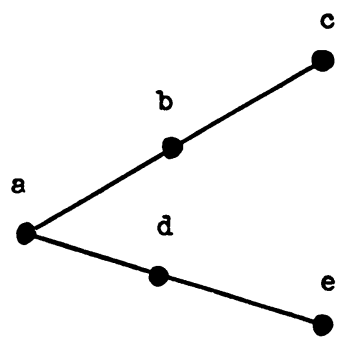

Because of Corollary 1.4.5, any $f \in \mathcal{S}^{+}(G)$ must take the fixed values which are circled below:

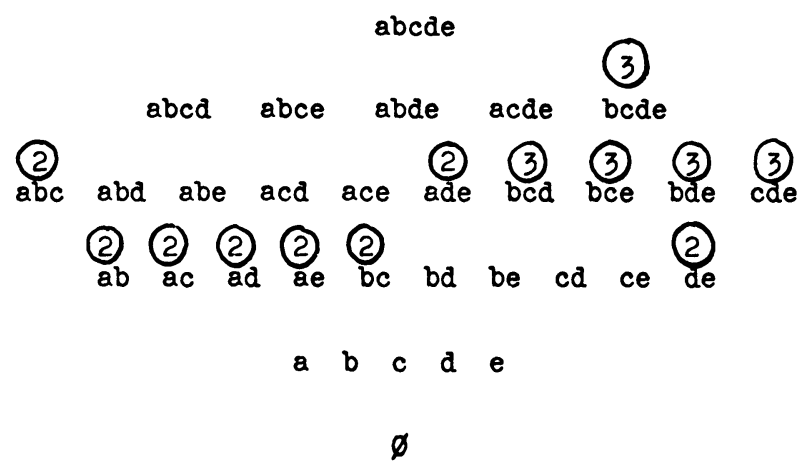

If we assign the values $1,2,2,2,2$ on $a, b, c, d, e$, a function $f_{1} \in \delta^{+}(G)$ is uniquely defined: 


$$
\mathbf{f}_{1} \equiv \begin{array}{llllllllll}
2 & 3 & 3 & 3 & 3 & 3 & 3 & & & \\
2 & 2 & 2 & 2 & 2 & 2 & 3 & 3 & 3 & 3 \\
& 1 & 2 & 2 & 2 & 2 & & 3 & 2 \\
& & & & 1 & & & & &
\end{array}
$$

To the values $2,1,2,2,2$ on $a, b, c, d, e$ corresponds uniquely $f_{2} \in \mathcal{S}^{+}(G)$ :

$$
\begin{aligned}
& 3 \\
& \begin{array}{lllll}
3 & 3 & 3 & 3 & 3
\end{array} \\
& f_{2} \equiv \begin{array}{llllllllll}
2 & 3 & 3 & 3 & 3 & 2 & 3 & 3 & 3 & 3 \\
2 & 2 & 2 & 2 & 2 & 3 & 3 & 3 & 3 & 2
\end{array} \\
& \begin{array}{lllll}
2 & 1 & 2 & 2 & 2
\end{array} \\
& 1
\end{aligned}
$$

$f_{1}$ and $f_{2}$ are two maximal elements of $\mathcal{S}^{+}(G)$ because no element of $\mathcal{S}^{+}(G)$ can take the values 2, 2, 2, 2, 2 on $a, b, c, d, e$ : we would have in that case:

$$
\begin{aligned}
f(a) & =f(a b)=f(a d)=2 \Rightarrow f(a b d)=2, \\
f(b) & =f(b c)=2 \Rightarrow f(b d)=f(b c d)=3, \\
f(b d) & >f(a b d), \text { contradiction. }
\end{aligned}
$$

In the cases of truncated Boolean algebras we have

PROPOSITION 1.4.10. If $G(r, S)$ is the truncated Boolean algebra on $S$ of rank $k<|S|$, the constant function $k$ on $B(S)$ is the maximal element of $\delta^{+}(G)$.

Proof. Clearly, the constant function $k$ on $B(S)$ belongs to $\mathcal{S}^{+}(G)$, because in $G(k, S)$ for any set $A,|A| \leqslant k$ is independent and $|A|>k$ is dependent.

Conversely, let $f \in \mathcal{S}^{+}(G): \forall A \subset S,|A|=k+1, A$ being a circuit of $G$,

$$
f(A)=|A|-1=k \text { and }|A|=k \Rightarrow f(A)=k .
$$

Then, $\forall A,|A|=k+2$, we can write, for $a, b \in A, a \neq b$,

$$
k \leqslant f(A) \leqslant f(A-a)+f(A-b)-f(A-a-b)=k,
$$

and, by induction, it is clear that

$$
\forall A \subset S, \quad k \leqslant|A| \leqslant|S|, \quad f(A)=k,
$$

and also

$$
\forall A \subset S, \quad|A|<k, \quad r(A) \leqslant f(A) \leqslant f(S)=k .
$$

The constant function $k$ is thus the maximal element of $\mathcal{S}^{+}(G)$.

2. Applications of expansions. The general problem of interest here is the following: given a certain decomposition defined on elements of $e_{s}$ we want to characterize the elements of $\mathcal{C}_{s}$ which are indecomposable. The two cases 
we will consider will be the convex decomposition and the sum decomposition.

2.1. Extremal rays of the cone of semimodular, nondecreasing functions. Given a finite set $S$, any real-valued function $f$ on $B(S)$ is determined by an element of $\mathbf{R}^{\mid{ }^{|S|}}$ (some arbitrary lexicographic ordering of the elements of $B(S)$ being fixed) consisting of the values of $f$ on subsets of $S$.

By Theorem 1.2.1, an element of $\mathbf{R}^{\mid{ }^{|S|}},(f(A))_{A \in B(S)}$, represents a function $f$ on $B(S)$ which is semimodular, nondecreasing if and only if $\forall X \subset S$, $x, y \in S$,

$$
\Delta f(X, x, y)=f(X \cup x)+f(X \cup y)-f(X)-f(X \cup x \cup y)>0 .
$$

Thus $e_{S}$ is the subset of $\mathbf{R}^{2^{|S|}}$ defined by those inequalities for all possible choices of $X, x$ and $y$ and is thus a convex polyhedron in $\mathbf{R}^{2^{|s|}}$.

It is clear that if $f \in \mathcal{C}_{s}$ and $k \in \mathbf{R}, k \geqslant 0$, then $k f \in \mathcal{C}_{s}$ : $\bigodot_{s}$ is a convex cone. Our problem is to characterize the elements of $\mathcal{C}_{s}$ which are not convex combinations of some other elements of $e_{s}$, i.e. the extremal rays of $e_{s}$. More precisely, we have

DEFinition 2.1.1. An element $x$ of $e_{S}$ is extremal if and only if the only way to write $x=x_{1}+x_{2}$ with $x_{1} \in \mathcal{C}_{S}, x_{2} \in \mathcal{C}_{S}$ is when $\exists t \in \mathbf{R}, x_{1}=t x$ and $x_{2}=(1-t) x$.

The following remarks allow us to restrict our attention to a smaller subset of $e_{s}$ when searching for all extremal elements of $e_{s}$.

(1) If $f \in \mathcal{C}_{s}$, for any real number $\lambda$, then $f+\lambda$ is also an element of $e_{s}: f$ is extremal if and only if the function $f-f(\varnothing)$ is extremal. Without loss of generality, we will consider normalized extremal elements of $C_{s}$ (i.e. $f(\varnothing)=$ 0).

(2) $e_{s}$ is defined by a finite number of inequalities: an extremal ray of $e_{s}$ is the solution of some system of equations consisting of a subset of those inequalities transformed into equalities: the values of an extremal function are the solution of a system of linear equations with integer coefficients are thus rational. Thus a function $f \in \mathcal{C}_{s}$ is extremal if and only if some integer multiple of $f$ is integer-valued: w.l.o.g. we can restrict our attention to integer-valued functions.

(3) Given $f \in \mathfrak{C}_{S}^{\circ}$, let $S^{\prime}$ be the support of $f$ :

$$
S^{\prime}=\{x \mid x \in S, f(x) \neq 0\},
$$

and let $f^{\prime}$ be the restriction of $f$ to $B\left(S^{\prime}\right)$. Clearly $f$ is completely determined by $f^{\prime}$ :

if $A \subset S^{\prime}, \quad f(A)=f^{\prime}(A)$,

if $A \not \subset S^{\prime}, \quad f(A)<f\left(A \cap S^{\prime}\right)+f\left(A-S^{\prime}\right)$,

$\forall A \subset S$. 
But $\forall a \in A-S^{\prime}, f(a)=0$, so $f\left(A-S^{\prime}\right) \leqslant \Sigma_{a \in A-S^{\prime}} f(a)=0$ and $f(A-$ $\left.S^{\prime}\right)=0$. Thus $f(A) \leqslant f\left(A \cap S^{\prime}\right)=f^{\prime}\left(A \cap S^{\prime}\right)$.

Finally $f$ is determined by $\forall A \subset S, f(A)=f^{\prime}\left(A \cap S^{\prime}\right)$. Furthermore, $f$ is extremal if and only if $f^{\prime}$ is extremal.

As a result of those remarks, without loss of generality, we will only consider extremal elements of $\mathcal{C}_{s}$ which are integer-valued, normalized and positive on points.

The following proposition gives another, often useful, definition of extremality for an element of a convex cone.

Proposition 2.1.2. $C$ being a convex cone in $\mathbf{R}^{n}$, an element $x \in C$ is not extremal if and only if $\exists \varepsilon \in \mathbf{R}^{n}, \varepsilon \neq 0$ and $\varepsilon$ not proportional to $x$, such that $x+\varepsilon \in C$ and $x-\varepsilon \in C$.

PRoof. If $x$ is extremal, and if $\exists \varepsilon \in \mathbf{R}^{n}$ such that $x+\varepsilon \in C$ and $x-\varepsilon \in$ $C$, then $x=\frac{1}{2}(x+\varepsilon)+\frac{1}{2}(x-\varepsilon) \Rightarrow \exists t \in \mathbf{R}, x-\varepsilon=2 t x$ and $x+\varepsilon=2 t x$, so $\varepsilon=(1-2 t) x: \varepsilon$ is either 0 or proportional to $x$.

Conversely if $x$ is not extremal, $\exists x_{1}, x_{2} \in C$ such that $x=x_{1}+x_{2}$. Let $\varepsilon=x_{1} ;$ then $x+\varepsilon=2 x_{1}+x_{2} \in C ; x-\varepsilon=x_{2} \in C$.

We will often determine the extremality of a given element $f \in I \varrho_{S}^{\circ}$ by trying to find a real-valued function $\varepsilon$ such that $f+\varepsilon \in I \bigodot_{S}^{o}$ and $f-\varepsilon \in$ $I e_{s}^{o}$. We have to solve the system of inequalities:

$$
\begin{aligned}
& \Delta(f+\varepsilon)(X, x, y) \geqslant 0, \\
& \forall X \subset S, \quad x, y \in S, \\
& \Delta(f-\varepsilon)(X, x, y) \geqslant 0 .
\end{aligned}
$$

LEMMA 2.1.3. If for some $X, x, y$ we have $\Delta f(X, x, y)=0$, then necessarily $\Delta \varepsilon(X, x, y)=0$.

Proof. We want to have $\Delta(f+\varepsilon)(X, x, y) \geqslant 0$ :

$$
\Delta(f+\varepsilon)(X, x, y)=\Delta f(X, x, y)+\Delta \varepsilon(X, x, y)=\Delta \varepsilon(X, x, y) \geqslant 0
$$

and also $\Delta(f-\varepsilon)(X, x, y) \geqslant 0$,

$$
\Delta(f-\varepsilon)(X, x, y)=-\Delta \varepsilon(X, x, y) \geqslant 0 .
$$

As a first step we will concern ourselves with the smaller class of geometric rank-functions, i.e. elements $f$ of $I \mathfrak{C}_{S}^{\infty}$ such that $f(a) \leqslant 1$ for any $a \in S$. The definition of such a function $r$ on $S$ determines a pregeometry on $S, G(r, S)$, and we will show that extremality of $r$ is equivalent to connectedness of $G(r, S)$.

The following result is well known: Given a pregeometry $G(r, S)$,

(i) the relation $\Re$ defined among elements of $S$ by " $a, b \in S, a \Re b \Leftrightarrow$ there is a circuit of $G$ containing $a$ and $b$ " is an equivalence relation;

(ii) the equivalence classes are the separators of $G$. 
We will use an equivalent formulation for defining $\mathcal{R}$.

LEMMA 2.1.4. $a, b \in S, a \Re b \Leftrightarrow$ there is $a$ basis $B$ of $G$ such that $a \in B$ and $B-\{a\} \cup\{b\}$ is a basis of $G$.

Proof. If there is a circuit $K, a, b \in K$, then we can complete $K-b$ into a basis $B$ of $G: B=(K-b) \cup C$ with $C \cap(K-b) \neq \varnothing$. Then

$$
r[(B-a) \cup b]=r[(K-a) \cup C]=r(K \cup C)=r(B)
$$

and $(B-a) \cup b$ is a basis.

Conversely, let $a, b \in S$ such that there is a basis $B$ and $a \in B,(B-a) \cup$ $b$ is a basis. $B \cup b$ is dependent: $b$ belongs to a circuit $K, K \subset B \cup b$. If $a \notin K$, then $K \subset(B-a) \cup b$, which is impossible, as $(B-a) \cup b$ is a basis, so $a \in K$ and thus $a \Re b$.

We will use the following notation: to each point $a$ of $S$, we associate a real variable $x_{a}$ and for any family of subsets of $S,\left(A_{i}\right)_{i \in I},\left(\sum_{a \in A_{i}} x_{a}=\right)_{i \in I}$ will stand for the system of equations

$$
\sum_{a \in A_{1}} x_{a}=\sum_{a \in A_{2}} x_{a}=\cdots=\sum_{a \in A_{i}} x_{a}=\cdots \cdot
$$

The main result of this section is

THEOREM 2.1.5. Given a pregeometry $G(r, S)$ whose family of bases is $\mathscr{B}=\left(B_{i}\right)_{i \in I}$, the following properties are equivalent:

(i) $G(r, S)$ is connected;

(ii) the linear system of equations $\left(\sum_{a \in B_{i}} x_{a}=\right)_{i \in I}$ is of dimension $|S|-1$;

(iii) the rank-function $r$ of $G$ is an extremal element of $I \bigodot_{S}^{o}$.

Proof. (i) $\Rightarrow$ (ii). $G$ being connected, by Lemma 2.1 .4 , the equivalence relation $\Re$ defines only one equivalence class: $\forall p, q \in S, \exists B \in \mathscr{B}$ such that $p \in B$ and $(B-p) \cup q \in \mathscr{B}$. Thus in the system $\Sigma \equiv\left(\Sigma_{a \in B_{i}} x_{a}=\right)_{i \in I}$, we have the equation

$$
\sum_{a \in B} x_{a}=\sum_{a \in(B-p) \cup q} x_{a} \text {, i.e. } x_{p}=x_{q}
$$

Thus $\Sigma$ has a solution, $\forall a, b \in S, x_{a}=x_{b}$, unique up to a multiplicative constant: $\Sigma$ being a system of homogeneous equations, this means that $\Sigma$ is of dimension $|S|-1$.

(ii) $\Rightarrow$ (iii). $G$ being given with its rank-function $r$, we want to look for a function $\varepsilon: B(S) \rightarrow \mathbf{R}$ such that $r \pm \varepsilon \in \bigodot_{S}^{o}$.

By Lemma 2.1.3, we know that for any $X \subset S, x, y \in S$ such that $\Delta r(X, x, y)=0$, we must also have $\Delta \varepsilon(X, x, y)=0$. In particular, let $B=$ $\left\{b_{1}, b_{2}, \ldots, b_{k}\right\}$ be a basis of $G$ : 


$$
\begin{gathered}
r\left(\left\{b_{1}, b_{2}\right\}\right)=r\left(b_{1}\right)+r\left(b_{2}\right) \Rightarrow \varepsilon\left(\left\{b_{1}, b_{2}\right\}\right)=\varepsilon\left(b_{1}\right)+\varepsilon\left(b_{2}\right), \\
\ldots \\
r\left(\left\{b_{1}, b_{2}, \ldots, b_{k}\right\}\right)=r\left(b_{k}\right)+r\left(\left\{b_{1}, b_{2}, \ldots, b_{k-1}\right\}\right) \\
\Rightarrow \varepsilon\left(\left\{b_{1}, b_{2}, \ldots, b_{k}\right\}\right)=\varepsilon\left(b_{k}\right)+\varepsilon\left(\left\{b_{1}, \ldots, b_{k-1}\right\}\right),
\end{gathered}
$$

and, thus, for any basis $B=\left\{b_{1} b_{2} \cdots b_{k}\right\}$ of $G$,

$$
\varepsilon(B)=\sum_{b_{i} \in B} \varepsilon\left(b_{i}\right)
$$

On the other hand, let $B_{1}$ and $B_{2}$ be two bases of $G$ : there is a maximal chain $B_{1}=S_{0} \subset S_{1} \subset S_{2} \subset \cdots \subset S_{l}=S$ so that

$$
r\left(B_{1}\right)=r\left(S_{1}\right)=r\left(S_{2}\right)=\cdots=r(S) \Rightarrow \varepsilon\left(B_{1}\right)=\varepsilon\left(S_{1}\right)=\varepsilon(S) .
$$

For the same reason, $\varepsilon\left(B_{2}\right)=\varepsilon(S)$ and, finally, for any two bases of $G, B_{1}$ and $B_{2}$, we must have $\varepsilon\left(B_{1}\right)=\varepsilon\left(B_{2}\right)$.Thus, the function $\varepsilon$ must verify the system $\left(\sum_{a \in B_{i}} \varepsilon(a)=\right)_{i \in I}$ which is $\Sigma$ : $\Sigma$ being of dimension $|S|-1$, the system has a solution unique up to a multiplicative constant; the values $r(a)$, $a \in S$, being a solution, $\exists \lambda \in \mathbf{R}$ such that $\forall a \in S, \varepsilon(a)=\lambda r(a)$. We want to show that this condition implies that $\exists A \subset S, \varepsilon(A)=\lambda r(A)$.

For any independent set $A$ of $G$, clearly

$$
r(A)=\sum_{a \in A} r(a) \Rightarrow \varepsilon(A)=\sum_{a \in A} \varepsilon(a)=\sum_{a \in A} \lambda r(a)=\lambda r(A) .
$$

For any dependent set $A$, let $I_{A}$ be a maximal independent set of $A$ :

$$
r(A)=r\left(I_{A}\right) \Rightarrow \varepsilon(A)=\varepsilon\left(I_{A}\right)=\lambda r\left(I_{A}\right)=\lambda r(A) .
$$

Finally the only possible functions $\varepsilon$ such that $r \pm \varepsilon \in \bigodot_{S}^{o}$ are given by $\lambda r$, for any $\lambda \in \mathbf{R}$. Thus $r$ is an extremal element of $e_{S}^{o}$.

(iii) $\Rightarrow$ (i). If $r$ is an extremal element of $\mathcal{C}_{s}$, clearly $G(r, S)$ is connected: if $G$ is disconnected, say $G(r, S)=G_{1}\left(r_{1}, S_{1}\right) \oplus G_{2}\left(r_{2}, S_{2}\right)$, we have $r=r_{1}+r_{2}$, where $r_{1}$ and $r_{2}$ are not proportional to $r$, which is a contradiction.

The proof of Theorem 2.1.5 is thus complete.

The characterization of extremal rank-functions as rank-functions of connected pregeometries is a stepping stone for our solution to the general case. We will use the notion of expansions.

Given $f \in I C_{S}^{o}$, supposed positive on points of $S$, we will consider its expansions defined on the minimum set $X$ as described in $\$ 1.3$. For any expansion $g$ of $f$, we will call $\rho(g)$ the restriction of $g$ to the sub-Boolean algebra of $B(X)$ isomorphic to $B(S)$ (we thus have, by definition, $\rho(g)=f$ ). An element $f \in I \bigodot_{S}^{\infty}$ is said to be reduced if the only common divisor of its values is 1 .

Proposimion 2.1.6. $f$ being a reduced element of $I \mathcal{C}_{S}^{o}$, if $f$ has a disconnected expansion, then $f$ is not extremal. 
Proof. Let $g$ be a disconnected expansion of $f$ : then

$$
g=\sum_{i=1}^{k} g_{i} \text { and } \rho(g)=\sum_{i=1}^{k} \rho\left(g_{i}\right)=f .
$$

The $\rho\left(g_{i}\right)$ for $i=1, \ldots, k$ are not all proportional to one another and to $f: f$ is reduced and the $\rho\left(g_{i}\right), i=1, \ldots, k$, are integer valued; if the $\rho\left(g_{i}\right)$ are all proportional to $f$, we would have $\forall i, i=1, \ldots, k, \rho\left(g_{i}\right)=n_{i} f$, where $n_{i} \in \mathbf{N}$, $n_{i} \neq 0$, and, thus, $\sum_{i=1}^{k} n_{i} f=f$, which is impossible. So, $f$ is not extremal.

In order to have a characterization of extremal elements of $I \bigodot_{S}^{\infty}$ in terms of their expansions, we need

LEMMA 2.1.7. If a vector $v \in \mathbf{Z}^{n}$ can be written $v=\sum_{i=1}^{k} t_{i} v_{i}$, where $t_{i} \in \mathbf{R}$, $t_{i}>0, v_{i} \in \mathbf{Z}^{n}$, then there is a family of positive rational numbers $q_{1}, q_{2}, \ldots, q_{k}$ such that $v=\sum_{i=1}^{k} q_{i} v_{i}$.

PROOF. Let us consider $\sum_{i=1}^{k} x_{i} v_{i}=v$ as a system of linear equations where the $x_{i}$ 's are the unknowns. By hypothesis, it has a solution: $x_{i}=t_{i}, i=$ $1, \ldots, k$. We can set a number of unknowns as independent variables and uniquely solve the others in terms of those: say $x_{1} x_{2} \cdots x_{l}$ are the independent variables. By Cramer's rule, $\forall i, l \leqslant i \leqslant k, x_{i}=x_{i}\left(x_{1} x_{2} \ldots x_{l}\right)$ is a linear combination of $x_{1} x_{2} \ldots x_{l}$ with rational coefficients. By continuity of those functions, there is a neighborhood $N$ in $\mathbf{R}^{\prime}$ of $\left(t_{1}, t_{2}, \ldots, t_{l}\right)$ such that $\left(x_{1}, x_{2}, \ldots, x_{l}\right) \in N$; we have $x_{i}\left(x_{1}, \ldots, x_{l}\right)>0$ for $l \leqslant i \leqslant k$. $\mathbf{Q}^{\prime}$ being dense in $\mathbf{R}^{l}$, we can take a rational set of values in $N .\left(q_{1} q_{2} \cdots q_{l}\right)$ and then $q_{i}=x_{i}\left(q_{1} q_{2} \ldots q_{l}\right)$ are rational and positive. Writing back in the given form, $v=\sum_{i=1}^{k} q_{i} v_{i}$, we complete the proof of the lemma.

PRoposition 2.1.8. If an element $f$ of $I \mathfrak{C}_{S}^{0}$ is not extremal, some integer multiple of $f$ has a disconnected expansion.

Proof. Suppose $f \in I e_{S}^{o}$ is not extremal; then we can write $f=\sum_{i=1}^{k} t_{i} f_{i}$ where $f_{i} \in I e_{S}^{\circ}$ and $t_{i} \in \mathbf{R}, t_{i}>0$. By Lemma 2.1 .7 we can also write

$$
f=\sum_{i=1}^{k} q_{i} f_{i} \text { where } q_{i} \in \mathbf{Q}, q_{i}>0 .
$$

Thus for some integer $n$, we have

$$
n f=\sum_{i=1}^{k} f_{i}^{\prime} \quad \text { where } f_{i}^{\prime} \in I \mathfrak{C}_{S}^{o} .
$$

Then we expand arbitrarily each $f_{i}^{\prime}$, getting a pregeometry $G\left(r_{i}, S_{i}\right)$. By taking the direct sum of those pregeometries, we get $G(r, S)=\bigoplus_{i=1}^{k} G\left(r_{i}, S_{i}\right)$ which is a disconnected expansion of $n f$.

Note that in the above proof, $G(r, S)$ is a disconnected pregeometry and 
the components $G_{i}\left(r_{i}, S_{i}\right)$ are such that the functions $\rho\left(r_{i}\right)=f_{i}^{\prime}, i=1, \ldots, k$, are not all proportional to $f$. We will say that an element $f$ of $I \varrho_{s}^{\infty}$ has a proper disconnected expansion if $f$ has a disconnected expansion whose components are not all expansions of functions proportional to $f$.

As a consequence of Propositions 2.1.6 and 2.1.8, we have

THEOREM 2.1.9. An element $f$ of $I \mathfrak{C}_{s}^{\circ}$ is not extremal if and only if some integer multiple of $f$ has a proper disconnected expansion.

We now turn to the case of another decomposition, the sum decomposition defined on the subset of $\mathcal{C}_{s}$ constituted by all the rank-functions defined on $S$.

2.2. Sum of pregeometries. Given $k$ pregeometries $G_{i}\left(r_{i}, S\right)$, we recall the definition of their sum $S\left(G_{1}, G_{2}, \ldots, G_{k}\right)$ as being the pregeometry defined on $S$ whose independent sets are all sets $A \subset S$ such that

$$
\forall A^{\prime} \subset A, \quad\left|A^{\prime}\right| \leqslant \sum_{i=1}^{k} r_{i}\left(A^{\prime}\right) .
$$

Another equivalent definition is given in [10]: a set $A$ is independent in $S\left(G_{1}, G_{2}, \ldots, G_{k}\right)$ if and only if $A$ can be written $A=\cup_{i=1}^{k} A_{i}$, where $A_{i}$ is independent in $G_{i}, i=1, \ldots, k$.

The problem of interest to us is to determine pregeometries which can be expressed as the sum of other pregeometries; they will be called decomposable. A rank-function is decomposable if it is the rank-function of a decomposable pregeometry.

Given $G(r, S), \mathcal{S}(G)\left(\mathcal{S}^{+}(G)\right)$ will denote, as in $\S 1.4$, the set of all elements of $I \bigodot_{S}\left(I \bigodot_{S}^{o}\right)$ whose geometrization is $G(r, S)$.

Suppose $G(r, S)$ is the sum of $G_{i}\left(r_{i}, S\right), i=1, \ldots, k$; then $f=\sum_{i=1}^{k} r_{i} \in$ $\delta^{+}(G)$ and $f$ has a disconnected expansion $E(f, X)$, namely the direct sum $G_{1}\left(r_{1}, S\right) \oplus G_{2}\left(r_{2}, S\right) \oplus \cdots \oplus G_{k}\left(r_{k}, S\right)$. It turns out that the condition is also sufficient.

THEOREM 2.2.1. A pregeometry $G(r, S)$ is decomposable if and only if $\varsigma^{+}(G)$ contains an element which has a disconnected expansion.

Proof. Only the sufficiency part is to be proved.

Let $f \in \delta^{+}(G), f$ having a disconnected expansion. Say $E(f, X)=$ $G_{1}\left(r_{1}, S_{1}\right) \oplus G_{2}\left(r_{2}, S_{2}\right) \oplus \cdots \oplus G_{k}\left(r_{k}, S_{k}\right)$. The restrictions $f_{i}$ of the different rank-functions $r_{i}, i=1, \ldots, k$, to the sub-Boolean algebra of $B(X)$ isomorphic to $B(S)$ give $f=\sum_{i=1}^{k} f_{i}$.

The theorem is proved by showing that

$$
G=G\left(f_{1}, S\right)=S\left(G\left(f_{1}, S\right), G\left(f_{2}, S\right), \ldots, G\left(f_{k}, S\right)\right),
$$

which is a result of the following lemma: 
LEMMA 2.2.2. Given $f_{1}, f_{2}, f_{3} \in I e_{s}^{o}$, let $r$ be the rank-function of $G\left(f_{1}+f_{2}\right.$, $S)$; then $G\left(f_{1}+f_{2}+f_{3}, S\right)=G\left(r+f_{3}, S\right)$.

Proof. Let $r_{1}$ be the rank-function of $G\left(f_{1}+f_{2}+f_{3}, S\right)$ and $r_{2}$ that of $G\left(r+f_{3}, S\right)$. By Theorem 1.14, $\forall A \subset S$,

$$
\begin{aligned}
r_{1}(A) & =\inf _{T \subset A}\left\{f_{1}(T)+f_{2}(T)+f_{3}(T)+|A-T|\right\}, \\
r_{2}(A) & =\inf _{T \subset A}\left\{r(T)+f_{3}(T)+|A-T|\right\} \\
& =\inf _{T \subset A}\left\{f_{3}(T)+|A-T|+\inf _{U \subset T}\left\{f_{1}(U)+f_{2}(U)+|T-U|\right\}\right\} .
\end{aligned}
$$

It is clear that $r_{2} \leqslant r_{1}$.

But now, suppose the inf for $r_{2}(A)$ is attained for some sets $\bar{U} \subset \bar{T} \subset A$ :

$$
\begin{aligned}
r_{2}(A) & =f_{3}(\bar{T})+|A-\bar{T}|+f_{1}(\bar{U})+f_{2}(\bar{U})+|\bar{T}-\bar{U}| \\
& =f_{1}(\bar{U})+f_{2}(\bar{U})+f_{3}(\bar{T})+|A-\bar{U}| \\
& \geqslant f_{1}(\bar{U})+f_{2}(\bar{U})+f_{3}(\bar{U})+|A-\bar{U}| \geqslant r_{1}(A),
\end{aligned}
$$

so finally $r_{1}=r_{2}$.

As a consequence, Theorems 1.4.8 and 2.2.1 determine a class of indecomposable pregeometries, namely the connected pregeometries $G$ such that $\left|\delta^{+}(G)\right|=2$. For example, the Fano plane is indecomposable and the cube in three-space is indecomposable.

An important property of the decomposition of a pregeometry $G(r, S)$ into a sum $G=S\left(G_{1}, G_{2}, \ldots, G_{k}\right)$ is given by

THEOREM 2.2.3. Given $n$ pregeometries $G_{i}\left(r_{i}, S\right), i=1, \ldots, n$, defined on a set $S$, if $G$ is the geometrization of the function $f=\sum_{i=1}^{n} r_{i}$, then for any $i$, $i=1, \ldots, n, G_{i}\left(r_{i}, S\right)$ is a quotient of $G$.

Proof. $K$ is a circuit of $G$ if and only if

$$
\begin{aligned}
|K|-1=\sum_{i=1}^{n} r_{i}(K) \quad \text { (Proposition 1.4.1), } & \\
& \forall K^{\prime} \subset K, \quad K^{\prime} \neq K, \quad\left|K^{\prime}\right| \leqslant \sum_{i=1}^{n} r_{i}\left(K^{\prime}\right) .
\end{aligned}
$$

We need the following

LEMMA 2.2.4. If $K$ is a circuit of $G$, then $\forall x \in K, x \in \overline{K-x} G_{i}\left(r_{i}\right)$ for $i=1, \ldots, n$.

Proof. Suppose $\exists G_{i}\left(r_{i}\right)$ such that $x \notin \overline{K-x} G_{i}\left(r_{i}\right)$, say $i=1$. We have $r_{1}(K-x)=r_{1}(K)-1$ and 


$$
|K-x| \leqslant \sum_{i=1}^{n} r_{i}(K-x)<\sum_{i=1}^{n} r_{i}(K)=|K|-1
$$

which is a contradiction.

We proceed to prove Theorem 2.2.3. Let us consider the map $f_{i}: G \rightarrow G_{i}\left(r_{i}\right)$, $f_{i}$ being induced by the identity on $S$ : we want to show that $f_{i}$ is a strong map. Let $F$ be a flat of $G_{i}\left(r_{i}\right): f_{i}^{-1}(F)=F$. If $F$ is not a flat of $G, \exists x \in S$, $\exists K$ circuit of $G$ such that $K-x \subset F, x \notin F, x \in K$. But by Lemma 2.2.4, $x \in \overline{K-x} G_{i}\left(r_{i}\right)$, i.e., $x \in F$, which is a contradiction. Thus $f_{i}$ is a strong map and $G_{i}\left(r_{i}\right)$ is a quotient of $G$.

\section{BIBLIOGRAPHY}

1. T. H. Brylawski, A decomposition for combinatorial geometries, Trans. Amer. Math. Soc. 171 (1972), 235-282. MR 46 \#8869.

2. Modular constructions for combinatorial geometries, Trans. Amer. Math. Soc. 203 (1975), 1-44. MR 50 \#9631.

3. __ An affine representation for transversal geometries, SIAM 54 (1975), 143-160.

4. G. Choquet, Theory of capacities, Ann. Inst. Fourier (Grenoble) 5 (1953-1954), 131-295 (1955). MR 18, 295.

5. P. Crawley and R. P. Dilworth, Algebraic theory of lattices, Prentice-Hall, Englewood Cliffs, N.J., 1973.

6. H. H. Crapo, Single-element extensions of matroids, J. Res. Nat. Bur. Standards Sect. B 69B (1965), 55-65. MR 32 \#7461.

7. and its Applications, Dept. Statist., Univ. North Carolina, Chapel Hill, N.C., 1970, pp. 74-99. MR 42 \# 7536.

8. H. H. Crapo and G.-C. Rota, On the foundations of combinatorial theory: Combinatorial geometries, MIT Press, Cambridge, Mass., 1970. MR 45 \# 74.

9. T. A. Dowling and D. G. Kelly, Elementary strong maps between combinatorial geometries, University of North Carolina, Chapel Hill, N.C. (preprint).

10. J. Edmonds, Matroid partition, Mathematics of the Decision Sciences, Part I, Lectures in Appl. Math., vol. 11, Amer. Math. Soc., Providence, R.I., 1968, pp. 335-345. MR 38 \# 5654.

11. - Submodular functions, matroids, and certain polyhedra, Combinatorial Structures and their Applications, Gordon and Breach, New York, 1970, pp. 69-87. MR 42 \#5828.

12. J. Edmonds and G.-C. Rota, Submodular set functions (Abstract, Waterloo Combinatorics Conf.), Univ. of Waterloo, Ontario, 1966.

13. D. A. Higgs, $A$ lattice order on the set of all matroids on a set, Canad. Math. Bull. 9 (1966), 684-685.

14. __ Maps of geometries, J. London Math. Soc. 41 (1960), 612-618. MR 34 \# 1910.

15. __ Strong maps of geometries, J. Combinatorial Theory 5 (1968), 185-191. MR 38 \#89.

16. J. S. Pym and H. Perfect, Submodular functions and independence structures, J. Math. Anal. Appl. 30 (1970), 1-31. MR 41 \#8269.

17. J. Rosenmüller and H. G. Weidner, $A$ class of extreme convex set functions with finite carrier, Advances in Math. 10 (1973), 1-38. MR 47 \# 10086.

18. L. S. Shapley, Cores of convex games, Rand Corp., RM-4571-PR, 1965; RM-P-4620, 1971.

19. W. T. Tutte, Lectures on matroids, J. Res. Nat. Bur. Standards Sect. B 69B (1965), 1-47. MR 31 \#4023. 
20. D. J. A. Welsh, On matroid theorems of Edmonds and Rado, J. London Math. Soc. (2) 2 (1970), 251-256. MR 41 \#3309.

21. __ Related classes of set functions, Studies in Pure Math., Academic Press, London, 1971. MR 46 \#3348.

22. H. Whitney, On the abstract properties of linear dependence, Amer. J. Math. 57 (1935), 509-533.

Department of Mathematics, University of Montana, Missoula, Montana 59801 